\title{
Mechanisms of verapamil-enhanced chemosensitivity of gallbladder cancer cells to platinum drugs: Glutathione reduction and MRP1 downregulation
}

\author{
HAOLU WANG ${ }^{1}$, XINXING LI $^{1}$, TAO CHEN ${ }^{1}$, WEI WANG ${ }^{1}$, QIANG LIU ${ }^{2}$, HUI LI $^{3}$, JING YI ${ }^{3}$ and JIAN WANG ${ }^{1}$ \\ Departments of ${ }^{1}$ General Surgery, and ${ }^{2}$ Pathology, Renji Hospital, Shanghai Jiao Tong University School of Medicine, \\ Shanghai 200127; ${ }^{3}$ Department of Cell Biology, Key Laboratory of Cell Differentiation and Apoptosis of \\ the Ministry of Education, Institutes of Medical Sciences, Shanghai Jiao Tong University \\ School of Medicine, Shanghai 200025, P.R. China
}

Received October 1, 2012; Accepted November 8, 2012

DOI: $10.3892 /$ or.2012.2156

\begin{abstract}
Gallbladder cancer (GBC) is highly malignant with a low response rate after chemotherapy and platinum drugs are currently prominent in the treatment of biliary tract cancers. Therefore, the development of novel strategies to enhance the sensitivity of GBC to platinum drugs is required. In the present study, we examined the effects of verapamil, a classic chemosensitizer whose reported mechanisms of action include inhibiting the transport function of P-glycoprotein (MDR1) or stimulating glutathione $(\mathrm{GSH})$ transport by multidrug resistance-related protein 1 (MRP1), in combination with cisplatin (CDDP), carboplatin (CBP) or oxaliplatin on the GBC cell lines, SGC996 and GBC-SD. Our results demonstrated that the co-treatment with verapamil markedly enhanced the chemosensitivity of GBC cells in comparison with platinum drug treatment alone. The mechanisms involved included GSH reduction and MRP1 downregulation. Verapamil/CDDP co-treatment inhibited tumor xenograft growth via the downregulation of MRP1 expression. MRP1 was highly expressed in human GBC tissue compared to non-tumorous gallbladder tissue. Our data demonstrate that verapamil may be used as a safe chemosensitizer for platinum drugs in the treatment of
\end{abstract}

Correspondence to: Professor Jian Wang, Department of General Surgery, Renji Hospital, Shanghai Jiao Tong University School of Medicine, 1630 S. Dongfang Road, Shanghai 200127, P.R.China E-mail: dr_wangjian@yahoo.com.cn

Abbreviations: MDR, multidrug resistance; ABC, ATP-binding cassette; MRP, multidrug resistance-related protein; CDDP, cisplatin; CBP, carboplatin; GSH, glutathione; ROS, reactive oxygen species; MTT, 3-(4,5-dimethylthiazol-2-yl)-2,5-diphenyltetrazolium bromide; Annexin V-FITC, Annexin V-fluorescein isothiocyanate; PI, propidium iodide; DCFH-DA, 2,7-dichlorodihydrofluorescein diacetate; DCF, 2,7-dichlorofluorescein

Key words: gallbladder cancer, platinum, glutathione, multidrug resistance-related protein 1 , verapamil
GBC. It functions by ROS and ATP-binding cassette transporter-related mechanisms.

\section{Introduction}

Gallbladder cancer (GBC) is one of the most highly malignant carcinomas with a low curative resection rate (10-30\%) and a low response rate to chemotherapy $(20-40 \%)(1,2)$. Although $\mathrm{GBC}$ is non-sensitive to routine chemotherapy, platinum drugs have recently been proven effective for GBC treatment. The most notable advance in the chemotherapy of biliary tract cancers in the last 5 years is that compared with gemcitabine alone, cisplatin (CDDP) plus gemcitabine have improved the overall survival of patients by 3.6 months (3). Oxaliplatin plus gemcitabine have also shown promising results in patients with advanced biliary tract carcinoma $(4,5)$.

The most common mechanism of multidrug resistance (MDR) in cancer cells is frequently associated with the overexpression of 4 ATP-binding cassette (ABC) transporters: P-glycoprotein (MDR1), multidrug resistance-related protein 1 (MRP1), multidrug resistance-related protein 2 (MRP2), and the breast cancer resistance protein (ABCG2), which may cause the active efflux of a variety of anticancer drugs (6). MDR1, MRP1, MRP2 and ABCG2 in GBC contribute to the MDR phenotype in vitro and in vivo (7-9). The mechanisms of insensitivity to platinum drugs have been mainly referred to glutathione $(\mathrm{GSH})$ conjugation and the transportation of GSH conjugates of platinum drugs out of cancer cells (10). Low reactive oxygen species (ROS) levels, increased GSH levels or overexpressed GSH-related enzymes and pumps are observed in several drug-resistant cancer cells (11). We have previously found that the sensitivity of a number of cancer cell lines, such as $\mathrm{Du}-145$ (prostate carcinoma), to platinum drugs is $\mathrm{ABC}$ transporter-dependent and/or ROS-dependent $(8,12)$. Thus, we hypothesized that the inhibition of $\mathrm{ABC}$ protein expression or the suppression of GSH activity may enhance platinum cytotoxicity in GBC.

In order to improve the effect of chemotherapy, several chemosensitizers have been examined for GBC. Emodin (1,3,8-trihydroxy-6-methylanthraquinone; a type of natural 
anthraquinone enriched in the traditional Chinese herbal medicines) and somatostatin (a regulator of numerous gastrointestinal hormones) have been reported to enhance the chemosensitivity of GBC cells $(13,14)$. However, several of these chemosensitizers remain in the experimental stage (15). Verapamil, a calcium channel blocker, is a classic chemo-sensitizer which can enhance the antitumor effect of CDDP in several cancer cells including neuroblastoma and lung cancer in vitro or in vivo $(16,17)$. Its reported mechanisms include inhibiting the transport function of MDR1 or stimulating GSH transport by MRP1 $(18,19)$. Recently, verapamil has been confirmed to enhance antitumor efficacy in several clinical studies including metastatic colorectal cancer $(20,21)$; however, as regards GBC, its effects have not been examined in preclinical or clinical studies.

In the present study, we aimed to clarify the effects of verapamil on the sensitivity of GBC cells to platinum drugs and its mechanisms. We discovered that verapamil enhanced the anticancer efficacy of CDDP, carboplatin (CBP) and oxaliplatin to GBC cells both in vitro and in vivo without obvious systemic toxicity by decreasing GSH levels and inhibiting the expression of MRP1.

\section{Materials and methods}

Cells and reagents. The SGC996 human GBC cell line was provided by the Academy of Life Sciences, Tongji University (Shanghai, China) and the GBC-SD cell line was provided by the Department of General Surgery, Xinhua Hospital (Shanghai, China). SGC996 cells were maintained in RPMI-1640 medium (Gibco-BRL, Gaitherburg, MD, USA) and GBC-SD cells were maintained in DMEM medium (Gibco-BRL). These media were supplemented with $10 \%$ fetal bovine serum. Cells were cultured in a humidified atmosphere with $5 \% \mathrm{CO}_{2}$ at $37^{\circ} \mathrm{C}$. CDDP and CBP were obtained from Qilu Pharmaceutical Co., Ltd. (Jinan, China). Oxaliplatin was obtained from Jiangsu Hengrui Medicine Co., Ltd. (Lian Yungang, China). Verapamil was purchased from Sigma (St. Louis, MO, USA).

Cell viability and apoptosis analysis. Cells were seeded in 96-microculture-well plates $\left(1.5 \times 10^{4} / \mathrm{ml}\right.$ cells, $\left.100 \mu \mathrm{l} / \mathrm{well}\right)$ and cultured overnight before treatment. After exposure to the agents as indicated for 24 and $48 \mathrm{~h}$, cell viability was assayed using the 3-(4,5-dimethylthiazol-2-yl)-2,5-diphenyl-tetrazolium bromide (MTT) (Sigma) assay as previously described $(22,23)$.

Cells were plated in 6 -well plates $\left(5 \times 10^{5} / \mathrm{ml}\right.$ cells, $2 \mathrm{ml} /$ well $)$ and cultured overnight prior to treatment. After being treated with drugs for 24 and $48 \mathrm{~h}$, apoptotic rates were assessed by flow cytometry using the Annexin V-fluorescein isothiocyanate (Annexin V-FITC)/propidium iodide (PI) kit (BD Pharmingen, San Diego, CA, USA). All procedures were performed according to the manufacturer's instructions and analyzed by flow cytometry using a FACS Calibur (Becton-Dickson, San Diego, CA, USA) $(22,23)$.

Reverse transcriptase-polymerase chain reaction (RT-PCR). The expression levels of $M D R 1, A B C G 2, M R P 1$ and $M R P 2$ were monitored by RT-PCR. After being treated with drugs for $24 \mathrm{~h}$, SGC996 and GBC-SD cells were lysed with $1 \mathrm{ml}$ of
RNAse-clean TRIzol reagent (Invitrogen, Carlsbad, CA, USA), and processed according to the manufacturer's instructions to obtain total cellular RNA. The isolated total RNA (1 $\mu \mathrm{g})$ was reverse-transcribed using random primers and AMV Reverse Transcriptase (Promega, Madison, WI, USA) for $5 \mathrm{~min}$ at $70^{\circ} \mathrm{C}, 5 \mathrm{~min}$ on ice and $60 \mathrm{~min}$ at $37^{\circ} \mathrm{C}$. The single-stranded cDNA was amplified by polymerase chain reaction using GoTaq DNA polymerase (Promega). PCR of MDR1 gene was performed under the following conditions: $20 \mathrm{sec}$ at $94^{\circ} \mathrm{C}$, $30 \mathrm{sec}$ at $55^{\circ} \mathrm{C}$ and $60 \mathrm{sec}$ at $72^{\circ} \mathrm{C}$ for 35 cycles. The sequences for $M D R 1$ sense and antisense primers were 5'-CCCATCATT GCAATAGCAGG-3' and 5'-GTTCAAACTTCTGCTCC TGA-3', respectively. PCR analysis of the $A B C G 2$ gene was performed under the following conditions: $30 \mathrm{sec}$ at $94^{\circ} \mathrm{C}$, $30 \mathrm{sec}$ at $55^{\circ} \mathrm{C}$ and $30 \mathrm{sec}$ at $72^{\circ} \mathrm{C}$ for 30 cycles. The sequences for $A B C G 2$ sense and antisense primers were 5'-TGGCTG TCATGGCTTCAGTA-3' and 5'-GCCACGTGATTCTTCC ACAA-3', respectively. PCR analysis of the MRP1 and MRP2 genes was performed under the following conditions: $30 \mathrm{sec}$ at $94^{\circ} \mathrm{C}, 30 \mathrm{sec}$ at $58^{\circ} \mathrm{C}$ and $30 \mathrm{sec}$ at $72^{\circ} \mathrm{C}$ for 34 cycles. The sequences for $M R P 1$ sense and antisense primers were $5^{\prime}-\mathrm{TGG}$ TGGGCCTCTCAGTGTCTTA-3' and 5'-TCGGTAGCGC AGGCAGTAGTTC-3', respectively. The sequences for MRP2 sense and antisense primers were 5'-ATGCTTCCTGGGG ATAAT-3' and 5'-TCAAAGGCACGGATAACT-3', respectively. Equal amounts of RT-PCR products were loaded on $1.5 \%$ agarose gels. $\beta$-actin was used as the internal control. The sequences for $\beta$-actin sense and antisense primers were 5'-GA AGATGGTGATGGGGAT-3' and 5'-GAAGGTGAAGGTC GGAGC-3', respectively.

ABCG2 and MRP1 siRNA transfection. To determine the role of ABCG2 and MRP1 in cellular sensitivity to CDDP, ABCG2 or MRP1 siRNA oligonucleotides were transiently transfected, using the Lipofectamine 2000 reagent (Invitrogen) according to the manufacturer's instructions (Invitrogen) with modifications as previously described $(8,24)$. A non-specific siRNA was also transfected as the mock control. After 48 h, SGC996 cells were lysed for RT-PCR or exposed to CDDP for an additional $24 \mathrm{~h}$ prior to apoptosis assay. The siRNA sequences for ABCG2 were 5'-UAAUGAUGUCCAAGAAGAAGUCUGC-3' and 5'-GCAGACUUCUUCUUGGACAUCAUUA-3'; and for MRP1 were 5'-GGAGUGGAACCCCUCUCUG-3' and 5'-CAGAGAGGGGUUCCACUCC-3'.

ROS and GSH measurement. 2,7-Dichlorodihydrofluorescein diacetate (DCFH-DA) (Sigma) was used for ROS capture in the cells. The average fluorescence intensity of 2,7-dichlorofluorescein (DCF) stands for intracellular ROS levels $(8,22)$. The cultured cells were then exposed to the various drugs and $10 \mu \mathrm{M}$ of DCFH-DA at $37^{\circ} \mathrm{C}$ for $15 \mathrm{~min}$. After being washed once with ice-cold PBS, cells were harvested and maintained on ice for an immediate detection using the flow cytometer FACS Calibur.

The cells were treated with drugs for $12 \mathrm{~h}$ and then assayed according to the instructions of the GSH Assay kit (Jiancheng Bioengineering Institute, Nanjing, China) as previously described (8). All results obtained were normalized according to the cellular protein content, which was measured using the BCA protein assay kit purchased from Sigma. 
In vivo study in tumor-bearing mice. SGC996 cells were harvested, washed, resuspended in serum-free optimum medium and then injected subcutaneously into 6-week-old BALB/c-nu/nu mice, with $6 \times 10^{6}$ cells/mouse ( $\mathrm{n}=8$ mice/group, purchased from the Shanghai Experimental Animal Center, Shanghai, China). This experiment was undertaken after obtaining approval from the institutional committee. Three days after inoculation, the tumor-bearing mice were intraperitoneally administered normal saline, verapamil $(20 \mathrm{mg} / \mathrm{kg} / \mathrm{day})$, CDDP ( $2 \mathrm{mg} / \mathrm{kg} /$ day) and verapamil/CDDP, every 3 days. The mice were sacrificed after 18 days, and body weight, as well as tumor volume and weight were measured. The hearts, kidneys and livers of the mice were histologically examined to determine the systemic toxicity.

Immunohistochemistry for ABCG2 and MRPI expression. All tumors from the mice and surgical samples from 51 patients with GBC treated at the Department of General Surgery, Renji Hospital, Shanghai Jiao Tong University School of Medicine were collected for immunohistochemistry. A total of 15 nontumorous gallbladder samples obtained from patients with cholecystolithiasis that underwent cholecystectomy were also included for control in this assay. The protocols for the use of animals were approved by the Department of Laboratory Animal Sciences, Shanghai Jiao Tong University School of Medicine.

Immunohistochemistry was performed following the standard avidin/streptavidin-biotin peroxidase methods $(25,26)$. The tissue microarray slides ( $4 \mu \mathrm{M}$-slices) were deparaffinized, rehydrated and boiled for antigen retrieval $\left(30 \mathrm{~m}\right.$ at $98^{\circ} \mathrm{C}$ in citrate buffer pH 6.0). Primary antibodies against ABCG2 (1:150) and MRP1 (1:20) proteins (Abcam, USA) were used on the sections of the tumor tissue, with $1 \%$ BSA-PBS as the negative control. After being incubated overnight at $4^{\circ} \mathrm{C}$, the slides were incubated with biotinylated anti-mouse immunoglobulin (LSAB) for $30 \mathrm{~min}$ and then with horseradish peroxidase-conjugated streptavidin for $30 \mathrm{~min}$. Each step was followed by a washing with PBS. Staining was revealed by 3,3'-diaminobenzidine (DAB) and counterstained with hematoxylin.

Cells were scored on color micrographs of characteristic lesions and the mean count of DAB-positive staining intensity for ABCG2 or MRP1 signals was calculated using Adobe Photoshop CS.

Statistical analysis. Data are presented as the mean values \pm SE. SAS software was used for statistical analysis. ANOVA (analysis of variance) was applied for comparison of the means of 2 or multiple groups, in which the Student-Newman-Keuls (SNK) was further used for the comparison of 2 groups. A value of $\mathrm{P}<0.05$ was considered to indicate a statistically significant difference.

\section{Results}

Verapamil enhances platinum drug-induced inhibition of cell viability in GBC cells by increasing apoptosis. To examine the additive effect of verapamil on cell viability, the SGC996 and GBC-SD human GBC cells were treated with CDDP, CBP, oxaliplatin or verapamil alone, or co-treated. No obvious reduction in viable cell number was observed in the group treated with verapamil alone. Verapamil combination treatment with
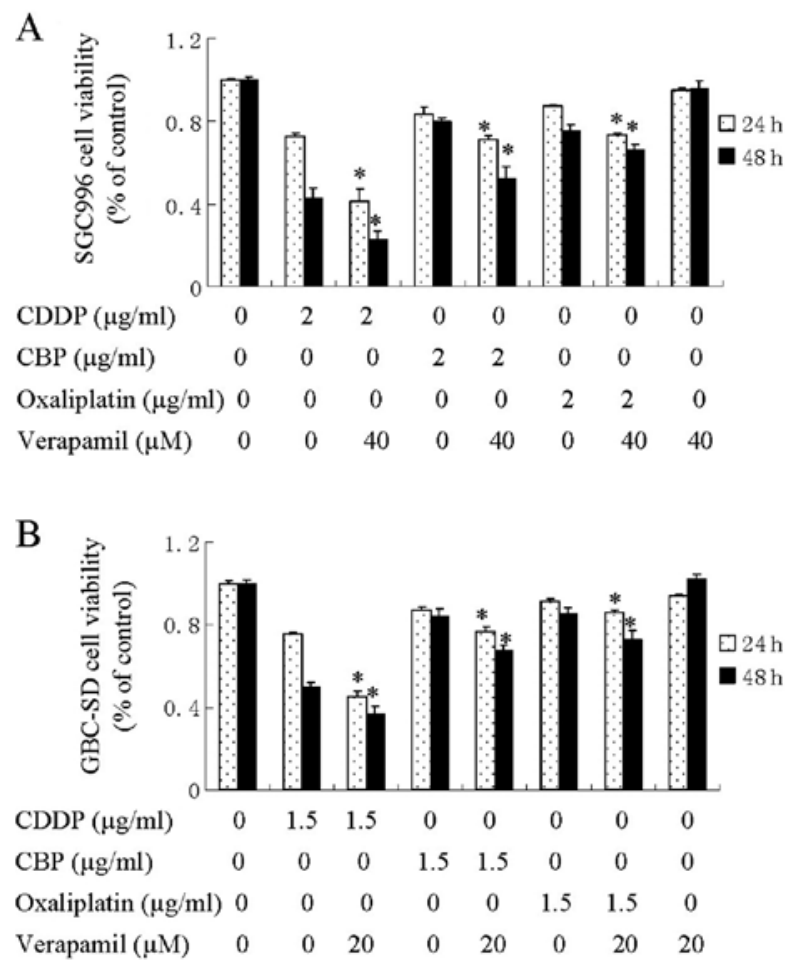

Figure 1. Cell viability of SGC996 and GBC-SD cells. Cells were exposed to CDDP, CBP, oxaliplatin and verapamil alone or CDDP, CBP and oxaliplatin/verapamil co-treatment. (A) Cell viability of SGC996 cells (MTT, drug treatments for 24 or 48 h). (B) Cell viability of GBC-SD cells (MTT, drug treatments for 24 or $48 \mathrm{~h}$ ). Columns, means of 3 experiments; bars, means $\pm \mathrm{SE}$. ${ }^{*} \mathrm{P}<0.05$, combination treatment group compared with the groups treated with CDDP, CBP and oxaliplatin alone.

CDDP, CBP and oxaliplatin led to a significant reduction in cell viability for 24 and $48 \mathrm{~h}$, compared with CDDP, CBP and oxaliplatin treatment alone (Fig. 1).

To determine whether the reduction in cell viability was attributed to the increase in apoptosis, Annexin V-FITC/PI double labeling flow cytometry was conducted. CDDP caused cell apoptosis, and verapamil enhanced the CDDP-induced apoptosis of the SGC996 (Fig. 2A-C) and GBC-SD cells (Fig. 2D-F) at 24 and $48 \mathrm{~h}$.

MRP1 is downregulated after verapamil/platinum drug co-treatment and is responsible for blockade of CDDP cytotoxicity. We investigated whether the enhanced apoptosis of GBC cells to platinum drugs/verapamil correlated with the regulation of the expression of the 4 MDR genes: MDR1, MRP1, MRP2 and ABCG2. The results from RT-PCR analysis demonstrated that the SGC996 cells expressed ABCG2, MRP1 and MRP2 at high levels; however, MDR1 expression was low, at an undetectable level. GBC-SD cells expressed ABCG2, MDR1 and MRP1 at high levels; however, MRP2 expression was undetectable (Fig. 3A).

Since verapamil enhanced the platinum drug-induced inhibition of cell viability in both GBC cell lines, we detected the co-expressed MDR genes in the 2 GBC cell lines, ABCG2 and MRP1. CDDP alone downregulated the expression of MRP1 and ABCG2 in the SGC996 cells, while CDDP/verapamil co-treatment resulted in a significant additive effect on the downregulation of the expression of MRP1, but not 
A

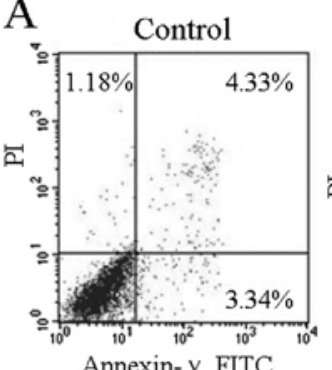

CDDP + Verapamil

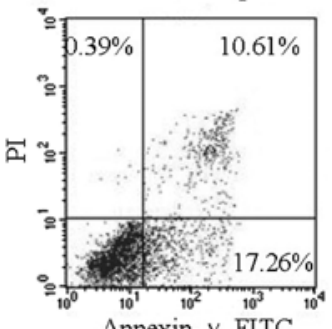

Annexin- $v$ FITC

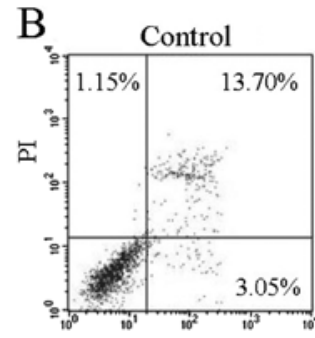

Annexin-v FITC

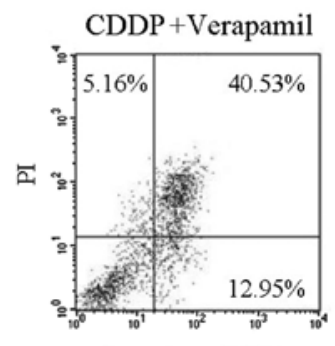

Annexin- $v$ FITC
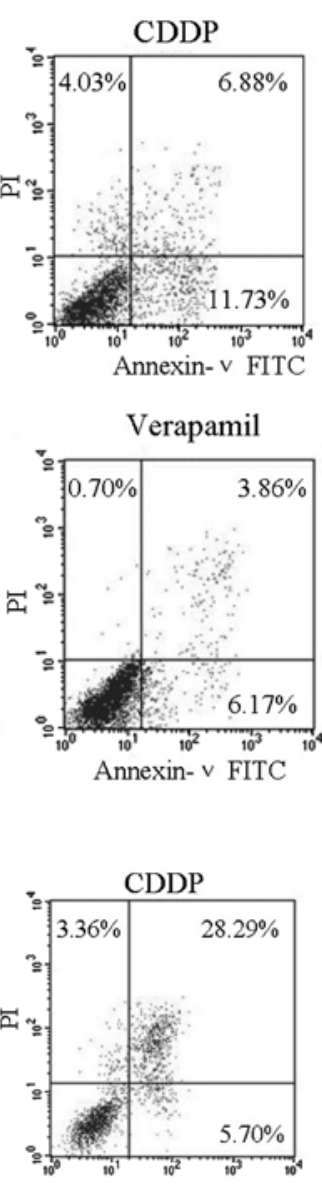

Annexin- $v$ FITC

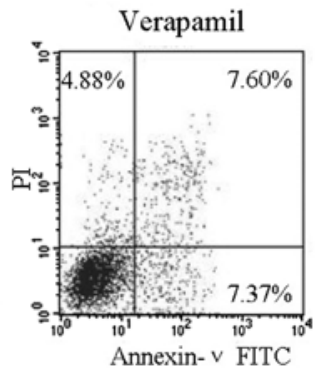

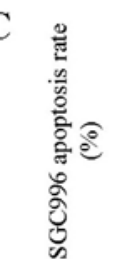

CDDP $(\mu \mathrm{g} / \mathrm{ml})$

Verapamil $(\mu \mathrm{M})$

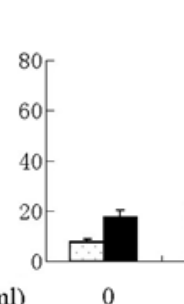

0

0

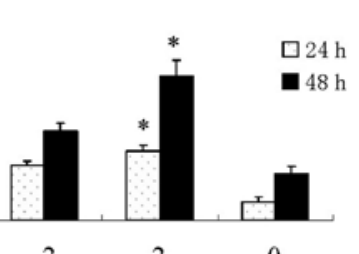

40

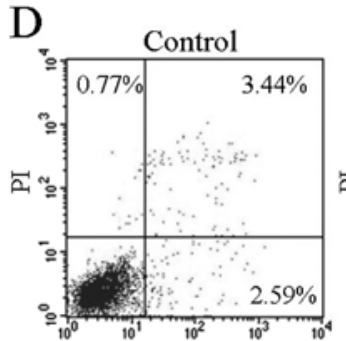

Annexin-v FITC

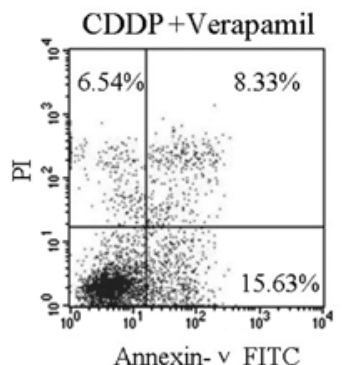

Annexin- $v$ FITC

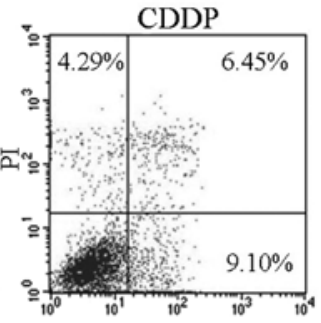

Annexin- $v$ FITC

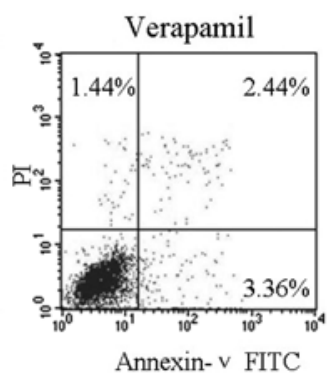

$\mathrm{E}$
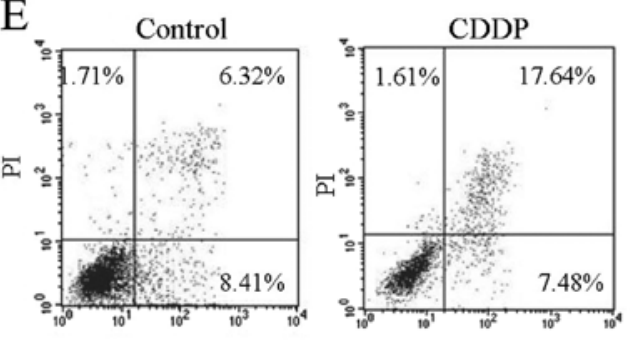

Annexin- $v$ FITC

Annexin- $v$ FITC

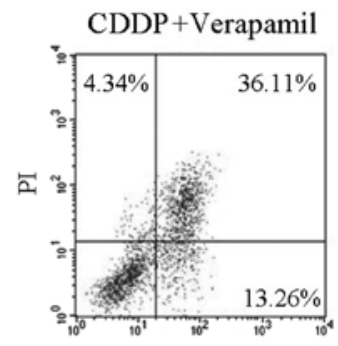

Annexin- $v$ FITC

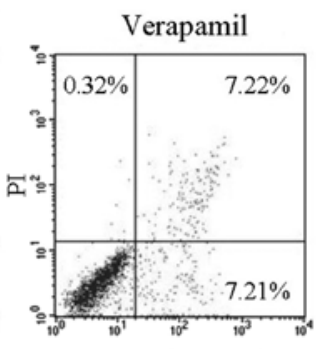

Annexin- $v$ FITC

F

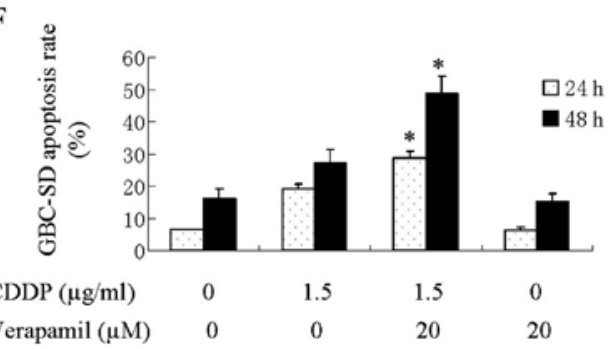

Figure 2. Apoptosis of SGC996 and GBC-SD cells. The apoptotic rate was analyzed by Annexin V/PI flow cytometry in SGC996 and GBC-SD cells treated with CDDP alone, verapamil alone and CDDP/verapamil co-treatment for 24 or $48 \mathrm{~h}$. (A) Apoptosis of SGC996 cells (drug treatments for $24 \mathrm{~h}$ ). (B) Apoptosis of SGC996 cells (48 h). (C) Analysis of apoptosis of SGC996 cells (Annexin V/PI flow cytometry, drug treatments for 24 or 48 h). (D) Apoptosis of GBC-SD cells (24 h). (E) Apoptosis of GBC-SD cells (48 h). (F) Analysis of apoptosis of GBC-SD cells (Annexin V/PI flow cytometry, drug treatments for 24 or 48 h). Density plots are shown in (A, B, D and E); bar charts are shown in (C and F). Columns, means of 3 experiments; bars, means \pm SE. ${ }^{*}<<0.05$, combination treatment group compared with the group treated with CDDP alone.

that of ABCG2. CBP/verapamil and oxaliplatin/verapamil slightly downregulated MRP1 expression in the SGC996 cells
(Fig. 3B). Co-treatment also downregulated MRP1 expression in GBC-SD cells, but ABCG2 did not (Fig. 3C). 
A

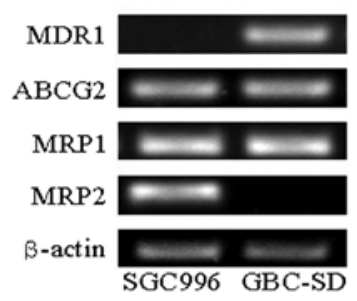

B

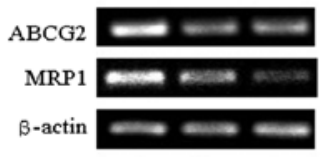

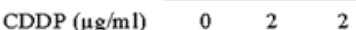
$\mathrm{CBP}(\mu \mathrm{g} / \mathrm{ml}) \quad 0 \quad 0 \quad 0$ Oxaliplatin $(\mu \mathrm{g} / \mathrm{ml}) \quad 0 \quad 0 \quad 0$ Verapamil $(\mu \mathrm{M}) \quad 0 \quad 0 \quad 40$

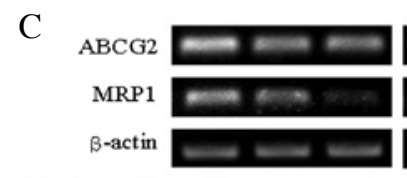

$\begin{array}{lccc}\operatorname{CDDP}(\mu \mathrm{g} / \mathrm{ml}) & 0 & 1.5 & 1.5 \\ \mathrm{CBP}(\mu \mathrm{g} / \mathrm{ml}) & 0 & 0 & 0 \\ \text { Oxaliplatin }(\mu \mathrm{g} / \mathrm{ml}) & 0 & 0 & 0 \\ \text { Verapamil }(\mu \mathrm{M}) & 0 & 0 & 20\end{array}$

$\mathrm{D}$
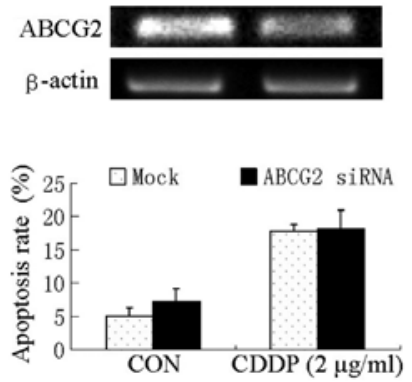

$\begin{array}{ccc}0 & 0 & 0 \\ 0 & 2 & 2 \\ 0 & 0 & 0 \\ 0 & 0 & 40\end{array}$

$\begin{array}{ccc}0 & 0 & 0 \\ 0 & 1.5 & 1.5 \\ 0 & 0 & 0 \\ 0 & 0 & 20\end{array}$

$\mathrm{E}$
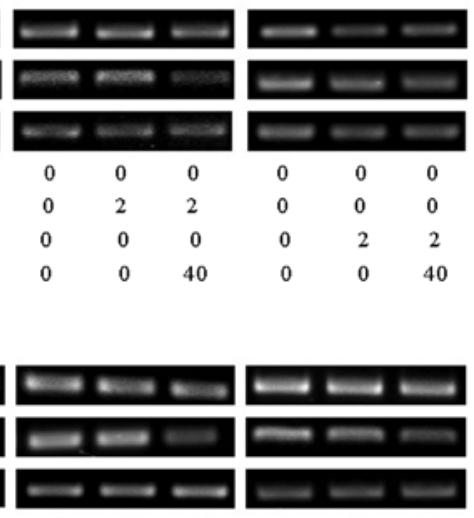

$0 \quad 1.5 \quad 1.5$

$1.5 \quad 1.5$

$0 \quad 0 \quad 20$

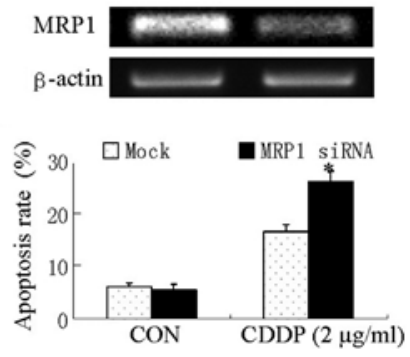

Figure 3. Expression of ABC transporters in SGC996 and GBC-SD cells. (A) Expression of MDR1, ABCG2, MRP1 and MRP2 in SGC996 and GBC-SD cells. (B) Expression of ABCG2 and MRP1 in SGC996 cells. SGC996 cells were exposed to CDDP, CBP or oxaliplatin alone, verapamil alone and CDDP, CBP or oxaliplatin/verapamil co-treatment for $24 \mathrm{~h}$ before being harvested for RT-PCR. (C) Expression of ABCG2 and MRP1 in GBC-SD cells. GBC-SD cells were exposed to CDDP, CBP or oxaliplatin alone, verapamil alone and CDDP, CBP or oxaliplatin/verapamil co-treatment for $24 \mathrm{~h}$ before being harvested for RT-PCR. (D) Apoptosis of SGC996 cells. Cells were transfected with non-specific siRNA (Mock) or ABCG2 siRNA for $48 \mathrm{~h}$. The apoptotic rate was analyzed by Annexin V/PI flow cytometry in SGC996 cells transfected with ABCG2 siRNA after being treated with CDDP for 24 h. (E) Apoptosis of SGC996 cells. Cells were transfected with non-specific siRNA (Mock) or MRP1 siRNA for $48 \mathrm{~h}$. The apoptotic rate was analyzed by Annexin V/PI by flow cytometry in SGC996 cells transfected with MRP1 siRNA after being treated with CDDP for $24 \mathrm{~h}$. Columns, means of 3 experiments; bars, means $\pm \mathrm{SE}$. ${ }^{*} \mathrm{P}<0.05$, MRP1 siRNA/CDDP group compared with the Mock/CDDP group.

To determine whether ABCG2 or MRP1 expression is responsible for the cytotoxic sensitivity of the SGC996 cells to CDDP, cells were transfected with siRNA oligonucleotide to silence MRP1 expression, and were then treated with CDDP for
$24 \mathrm{~h}$. The results demonstrated that the knockdown of ABCG2 had no significant effect (Fig. 3D), while the knockdown of MRP1 increased CDDP-induced cell apoptosis (Fig. 3E). These data suggest that MRP1 is responsible for CDDP resistance in GBC cells, and that verapamil may facilitate the cytotoxicity of CDDP by suppressing MRP1 expression.

Verapamil elevates intracellular ROS levels and decreases GSH levels of GBC cells. We found that the exposure of SGC996 and GBC-SD cells to verapamil or verapamil/platinum drugs resulted in a significant elevation of intracellular ROS levels, while platinum drug treatment alone had less of an effect (Fig. 4A and B). We measured the intracellular GSH levels after exposing SGC996 and GBC-SD cells to platinum drugs, verapamil, or platinum drugs in combination with verapamil for $12 \mathrm{~h}$. Verapamil alone or in combination with CDDP, $\mathrm{CBP}$ and oxaliplatin markedly reduced the intracellular GSH levels. These data indicated that the enhancement of verapamil on platinum drug-induced cytotoxicity in both GBC cell lines was related to the intracellular GSH reduction (Fig. 4C and D).

Verapamil enhances the sensitivity of tumor xenografts to $C D D P$ without systemic toxicity in vivo. In vitro experiments demonstrated that verapamil enhanced the sensitivity of gallbladder cancer cells to platinum drugs. To verify this effect in vivo and evaluate its side-effects, SGC996 cells were transplanted into nude mice and the mice were treated for 18 days. Our results demonstrated that tumor xenografts exposed to the combination therapy were significantly smaller compared to those from the other groups (Fig. 5A). Systemic toxic effects were evaluated by the body weight loss of mice and the pathological changes in major organs including the heart, kidney and liver. No notable differences were observed among these groups (Fig. 5B), demonstrating that verapamil/CDDP co-treatment had no obvious toxic effects in vivo.

Verapamil/CDDP co-treatment represses the expression of $M R P 1$ in tumors xenografts. To ascertain the effect of verapamil/CDDP co-treatment on ABCG2 and MRP1 expression in vivo, immunohistochemistry for $\mathrm{ABCG} 2$ and MRP1 expression was performed on paraffin-embedded tissue sections of tumors. The expression of ABCG2 in tumors was downregulated by CDDP; however, co-treatment with CDDP and verapamil did not lead to a more significant downregulation (Fig. 6A and B). The expression of MRP1 in tumors was downregulated by CDDP, and more significantly by verapamil/CDDP combination treatment (Fig. 6C and D). These data demonstrated that CDDP alone downregulated the expression of the MRP1 and ABCG2 proteins, while CDDP/verapamil co-treatment resulted in an additive effect on the downregulation of the expression of MRP1, but not that of ABCG2.

$A B C G 2$ and MRP1 are highly expressed in GBC tissue compared to non-tumorous gallbladder tissue. We used immunohistochemistry to determine the degree of ABCG2 and MRP1 expression in cancer and non-tumorous gallbladder tissue. The ABCG2 and MRP1 proteins were expressed predominantly in the membrane and partly in the cytoplasm of epithelial cells in both GBC and cholecystolithiasis specimens. There was stronger staining of ABCG2 and MRP1 in GBC 
A

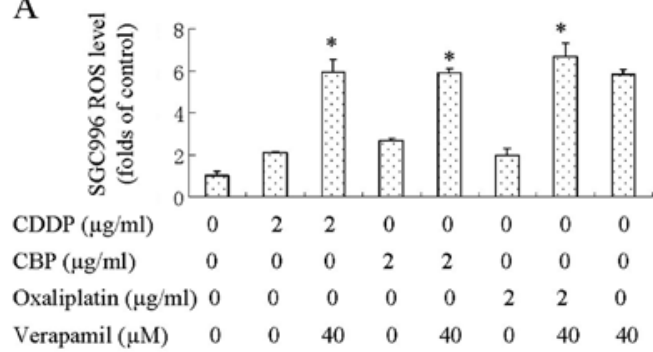

\section{B

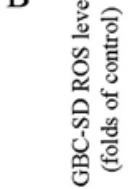

$\operatorname{CDDP}(\mu \mathrm{g} / \mathrm{ml})$

$\mathrm{CBP}(\mu \mathrm{g} / \mathrm{ml})$

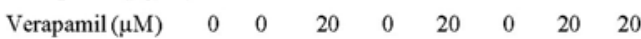

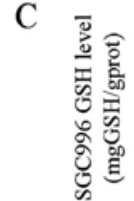

$\operatorname{CDDP}(\mu \mathrm{g} / \mathrm{ml})$

$\mathrm{CBP}(\mu \mathrm{g} / \mathrm{ml})$

Oxaliplatin $(\mu \mathrm{g} / \mathrm{ml})$

Verapamil $(\mu \mathrm{M})$
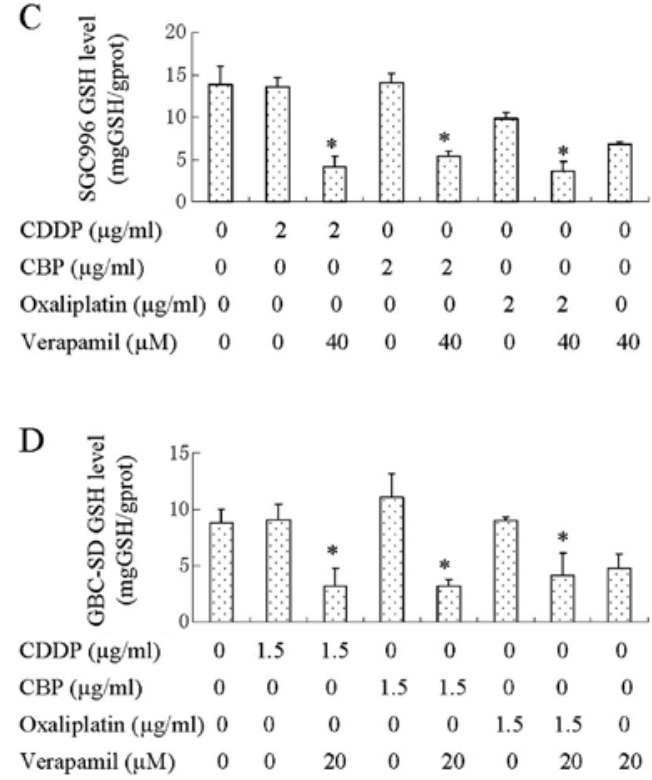

Figure 4. ROS and GSH in SGC996 and GBC-SD cells. Cells were exposed to CDDP, CBP, oxaliplatin or verapamil alone and CDDP, CBP, oxaliplatin/verapamil co-treatment. (A) ROS level in SGC996 cells (DCF flow cytometry, drug treatments for $15 \mathrm{~min}$ ). (B) ROS level in GBC-SD cells (DCF flow cytometry, drug treatments for $15 \mathrm{~min}$ ). (C) GSH levels in SGC996 cells (GSH analysis kit, drug treatments for $12 \mathrm{~h}$ ). (D) GSH levels in GBC-SD cells (GSH analysis kit, drug treatments for $12 \mathrm{~h}$ ). Columns, means of 3 experiments; bars, means $\pm \mathrm{SE}$. $\mathrm{P}<0.05$, combination treatment groups compared with the groups treated with CDDP, CBP and oxaliplatin alone.

compared to non-tumorous gallbladder tissue (signal intensity: $35.5 \pm 4.6$ vs. $17.1 \pm 11.2, \mathrm{P}<0.05 ; 40.4 \pm 18.1$ vs. $22.0 \pm 9.9, \mathrm{P}<0.05$ ) (Fig. 7).

\section{Discussion}

The 4 members of the ABC transporter family (MDR1, ABCG2, MRP1 and MRP2) play an important role in MDR in cancer cells $(6,7,9)$. In the present study, we investigated the expression of the $4 \mathrm{ABC}$ transporters in the $2 \mathrm{GBC}$ cell lines,
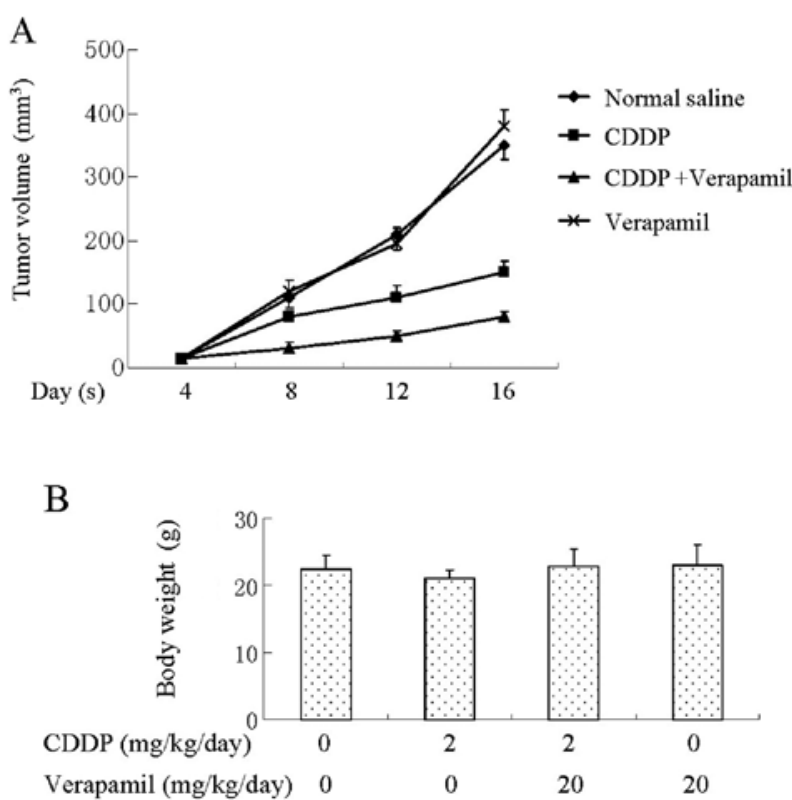

Figure 5. Growth capability of transplanted tumors formed by SGC996 cells and the toxic effects of therapy in tumor-bearing mice. The tumorbearing mice were injected intraperitoneally with normal saline, CDDP, verapamil/CDDP or verapamil. (A) Average volume of transplanted tumors. Columns, means; bars, means $\pm \mathrm{SE}$. ${ }^{*} \mathrm{P}<0.05$, combination treatment group compared with the group treated with CDDP alone $(n=8)$. (B) Average body weight of tumor-bearing mice $(n=8)$.

SGC996 (13) and GBC-SD (27), as well as in human tissue. We discovered that ABCG2 and MRP1 were highly expressed in the SGC996 and GBC-SD cells; while the expression of MDR1 in the SGC996 cells and that of MRP2 in the GBC-SD cells was undetectable. ABCG2 and MRP1 are also highly expressed in GBC tissue compared to non-tumorous tissue. Collectively, the results from previous reports $(8,9)$ and our present study allow us to hypothesize that ABCG2 and MRP1 are 2 common denominators of the intrinsic MDR phenotype in GBC cells. The accumulation of ABCG2 in poorly differentiated GBC may coincide with side population cells increasing and/or the malfunctioning of PI3K-signaling pathways during tumor progression $(9,28)$. ABCG2 and MRP1 overexpression has also been documented in other MDR cancer cells $(6,29)$.

Verapamil is a specific first-generation MDR1 inhibitor and differs from other GBC chemosensitizers, such as emodin or somatostatin. Its efficiency has been confirmed in certain types of cancer, both in preclinical studies and in clinical use. It has been reported that co-treatment with verapamilchemotherapeutic agents (including cisplatin) downregulates MDR1 gene transcription (17,30). We found that verapamil also enhanced the chemosensitivity of GBC cells (SGC996) in which the expression of MDR1 was undetectable. To the best of our knowledge, this is the first report demonstrating the suppressive effect of verapamil on MRP1 expression in cancer cells. A major fraction of the intracellular platinum drugs are conjugated with GSH to form less toxic GS-platinum complexes and are discharged from cancer cells via the glutathione conjugate export pumps, such as MRP1 $(10,31)$. In addition, we demonstrated that silencing MRP1 expression may increase CDDP-induced GBC cell apoptosis. These data support our hypothesis that MRP1 downregulation causes the 
A

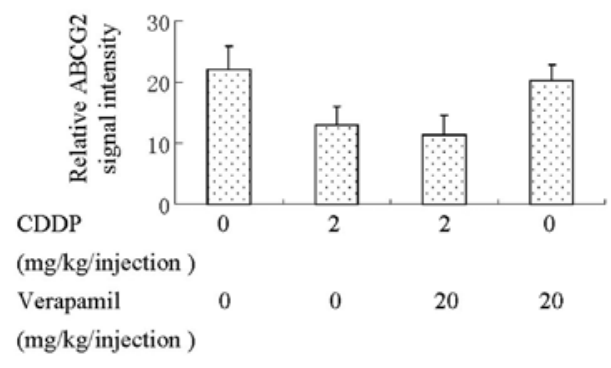

B

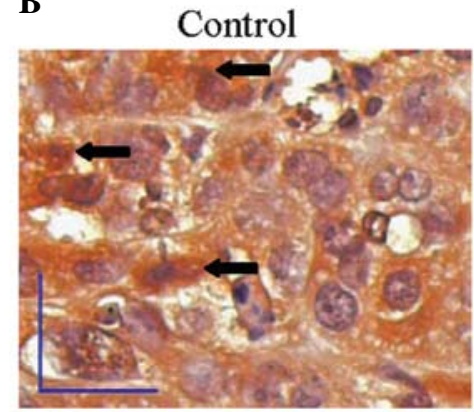

CDDP+Verapamil

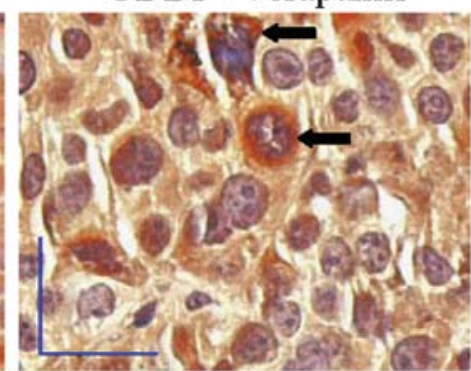

C

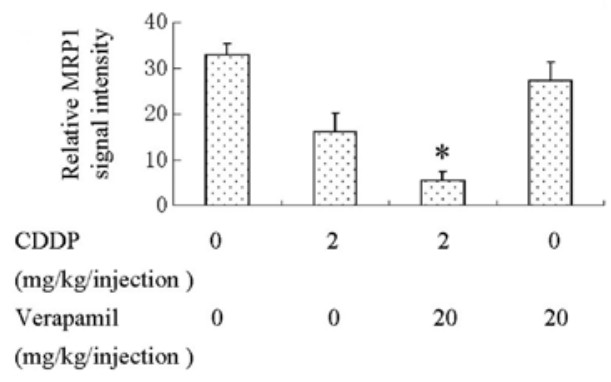

D

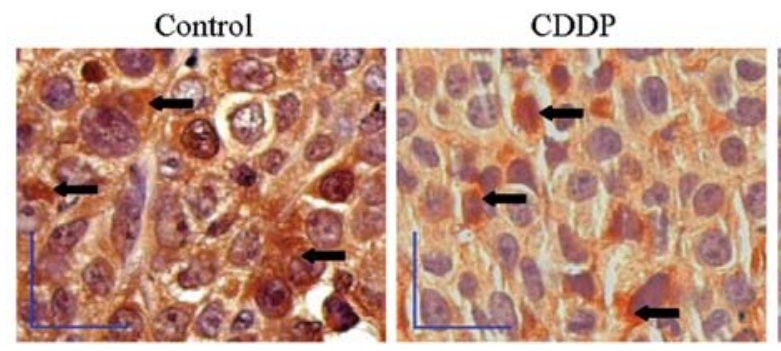

CDDP +Verapamil

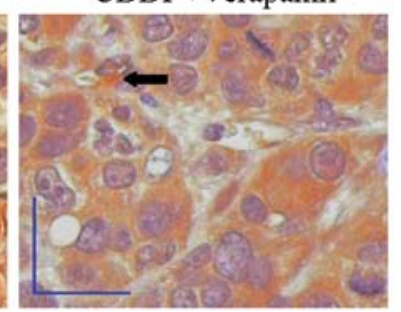

Figure 6. Expression of ABCG2 and MRP1 protein in transplanted tumor tissues. The cytoplasmic brown staining (arrows) represents a positive signal for the ABCG2 and MRP1 proteins. (A) Analysis of relative ABCG2 signal intensity of the 4 groups. (B) Expression of ABCG2 in the tumor tissues of the control and $\mathrm{CDDP} /$ verapamil group. Scale bar, $30 \mu \mathrm{m}$. (C) Analysis of relative MRP1 signal intensity of the 4 groups. (D) Expression of MRP1 in the tumor tissues of the control, CDDP and CDDP/verapamil groups. Scale bar, $30 \mu \mathrm{m}$. Columns, means of 3 experiments; bars, means $\pm \mathrm{SE}$. * $\mathrm{P}<0.05$, combination treatment group compared with the group treated with CDDP alone.

A

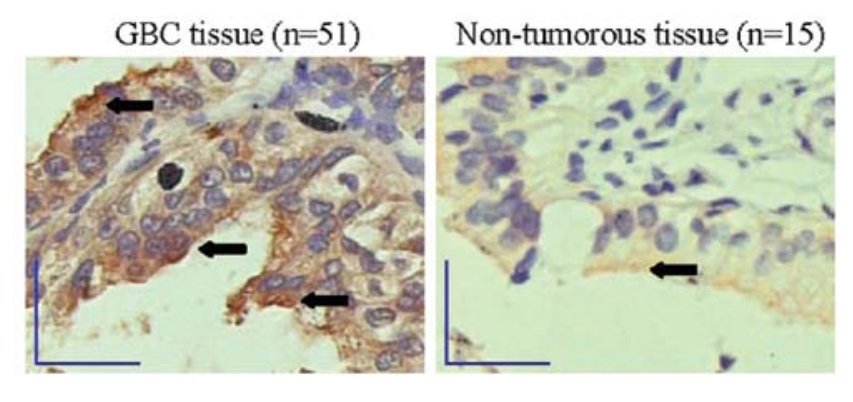

B

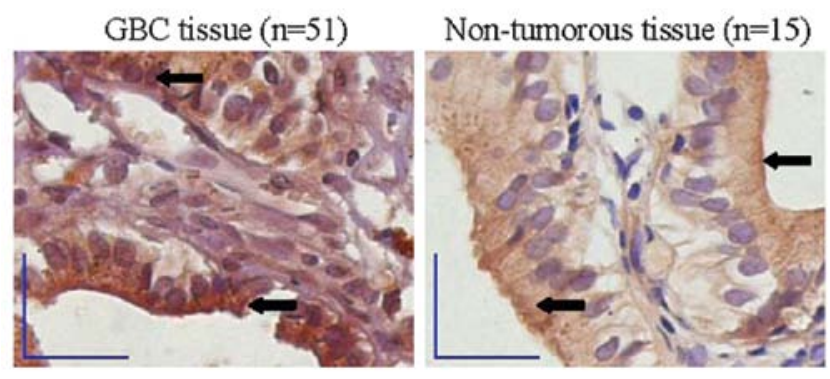

Figure 7. Expression of ABCG2 and MRP1 proteins in human GBC ( $\mathrm{n}=51)$ and non-tumorous tissues ( $\mathrm{n}=15)$. The cytoplasmic brown staining (arrows) represents a positive signal for ABCG2 and MRP1 proteins. (A) Expression of ABCG2 in human GBC and non-tumorous tissues. (B) Expression of MRP1 in human GBC and non-tumorous tissues. Scale bar, $30 \mu \mathrm{m}$.

accumulation of intracellular platinum drugs and enhances their cytotoxicity. Transcription factors, such as p53 and Sp1 may regulate the transcription of MRP1 $(32,33)$. The upstream regulators that may serve as the target of verapamil-platinum drug co-treatment to mediate the downregulation of MRP1 require clarification in future studies. MRP1 downregulation may be one of the mechanisms behind the verapamil-enhanced cytotoxicity of platinum drugs on GBC cells. Compared to CBP or oxaliplatin, CDDP inhibited GBC cell viability more significantly either alone or in combination with verapamil and it downregulated MRP1 more significantly. These findings may have relevant clinical implications explaining why CDDP is a superior choice to other platinum drugs in several clinical trials (3). Although with a much less significant effect on the downregulation of MRP1, CBP or oxaliplatin also had a notable inhibitory effect on GBC cell viability either alone or in combination with verapamil. Thus, other more important mechanisms to enhance cytotoxicity may be involved.

Depletion of intracellular GSH may restore the drug sensitivity of various cancer cells (34). Thus, the reduction in GSH levels in GBC cells may contribute to the enhanced cytotoxicity of platinum drugs. However, verapamil, which stimulates GSH transport by MRP1, has been reported to be only slightly, or not at all, effective in restoring the drug sensitivity of several MRP1-overexpressing cells $(35,36)$. Only several types of MRP1-overexpressing cells display hypersensitivity to verapamil, which triggers apoptosis through MRP1-mediated GSH extrusion $(37,38)$. In the present study, it is notable that verapamil enhanced the cytotoxicity of GBC cells to platinum drugs and a remarkable reduction in intracellular GSH levels 
followed the verapamil-platinum drug combination treatment. The enhancement of apoptosis cannot be achieved through the simple augmentation of ROS levels or a decrease in GSH levels, since a single administration of verapamil has no effect in vitro and in vivo. We suggest that intracellular GSH reduction in GBC cells and thereby less platinum conjugates formed with less cellular efflux of these conjugates may contribute to verapamil-induced increased cytotoxicity. Since CBP or oxaliplatin in combination with verapamil had a notable inhibitory effect on GBC cell viability but a much less significant effect on the downregulation of MRP1, verapamil is supposed to act primarily as a functional modulator of GSH rather than as a modifier of MRP1 expression. Our findings may at least partly explain the apparent controversial effect of verapamil on MRP1-associated drug resistance and reflect the entire complexity of MDR, since GSH reduction and MRP1 downregulation co-exist in reversing the MDR phenotype in cancer cells.

In conclusion, our data present evidence that verapamil may effectively enhance the anticancer effect of platinum drugs on GBC cells with little systemic toxic effects. Verapamil or more potent and safer verapamil analogs may be further developed in the frame of a new therapeutic strategies aimed at eliminating MDR in cancer cells with high MRP1 expression. In addition, we demonstrate that GSH reduction and MRP1 downregulation are the mechanisms involved in verapamil reversing the MDR phenotype in GBC cells. Understanding the complex mechanisms of MDR may lead to novel approaches in chemotherapy.

\section{Acknowledgements}

The authors thank Mrs. Yuying Chen, Mrs. Xiaojiao Huo and Mrs. Guiying Shi, Shanghai Jiao Tong University School of Medicine, for their technical assistance in this study. This study was supported by grants from the Shanghai Science and Technology Commission (09411960800, to J.W.).

\section{References}

1. Gourgiotis S, Kocher HM, Solaini L, Yarollahi A, Tsiambas E and Salemis NS: Gallbladder cancer. Am J Surg 196: 252-264 2008

2. Zhu AX, Hong TS, Hezel AF and Kooby DA: Current management of gallbladder carcinoma. Oncologist 15: 168-181, 2010.

3. Valle J, Wasan H, Palmer DH, et al: Cisplatin plus gemcitabine versus gemcitabine for biliary tract cancer. N Engl J Med 362 : $1273-1281,2010$.

4. Hollebecque A, Bouche O, Romano O, et al: Experience of gemcitabine plus oxaliplatin chemotherapy in patients with advanced biliary tract carcinoma. Chemotherapy 56: 234-238, 2010.

5. Andre T, Reyes-Vidal JM, Fartoux L, et al: Gemcitabine and oxaliplatin in advanced biliary tract carcinoma: a phase II study. Br J Cancer 99: 862-867, 2008.

6. Wu CP, Calcagno AM and Ambudkar SV: Reversal of ABC drug transporter-mediated multidrug resistance in cancer cells: evaluation of current strategies. Curr Mol Pharmacol 1: 93-105, 2008.

7. Cao L, Duchrow M, Windhovel U, Kujath P, Bruch HP and Broll R: Expression of MDR1 mRNA and encoding P-glycoprotein in archival formalin-fixed paraffin-embedded gall bladder cancer tissues. Eur J Cancer 34: 1612-1617, 1998.

8. Wang W, Sun YP, Huang XZ, et al: Emodin enhances sensitivity of gallbladder cancer cells to platinum drugs via glutathion depletion and MRP1 downregulation. Biochem Pharmacol 79: 1134-1140, 2010.
9. Aust S, Obrist P, Jaeger W, et al: Subcellular localization of the ABCG2 transporter in normal and malignant human gallbladder epithelium. Lab Invest 84: 1024-1036, 2004.

10. Suzuki T, Nishio K and Tanabe S: The MRP family and anticancer drug metabolism. Curr Drug Metab 2: 367-377, 2001.

11. Zhang K, Mack P and Wong KP: Glutathione-related mechanisms in cellular resistance to anticancer drugs. Int J Oncol 12: 871-882, 1998

12. Huang XZ, Wang J, Huang C, et al: Emodin enhances cytotoxicity of chemotherapeutic drugs in prostate cancer cells: the mechanisms involve ROS-mediated suppression of multidrug resistance and hypoxia inducible factor-1. Cancer Biol Ther 7: 468-475, 2008.

13. Wang W, Sun Y, Li X, et al: Emodin potentiates the anticancer effect of cisplatin on gallbladder cancer cells through the generation of reactive oxygen species and the inhibition of survivin expression. Oncol Rep 26: 1143-1148, 2011.

14. Quan ZW, Yang Y, Li JY, Gong W, Qin YY and Li SG: The mechanisms of somatostatin induced enhanced chemosensitivity of gallbladder cancer cell line to doxorubicin: cell cycle modulation plus target enzyme up-regulation. Biomed Pharmacother 64: 451-457, 2010.

15. Zhang JT, Fan YZ, Chen CQ, Zhao ZM and Sun W: Norcantharidin: a potential antiangiogenic agent for gallbladder cancers in vitro and in vivo. Int J Oncol 40: 1501-1514, 2012.

16. Ikeda H, Nakano G, Nagashima K, et al: Verapamil enhancement of antitumor effect of cis-diamminedichloroplatinum(II) in nude mouse-grown human neuroblastoma. Cancer Res 47: 231-234, 1987.

17. Wang J, Wang H,Zhao L, et al: Down-regulation of P-glycoprotein is associated with resistance to cisplatin and VP-16 in human lung cancer cell lines. Anticancer Res 30: 3593-3598, 2010.

18. Perrotton T, Trompier D, Chang XB, Di Pietro A and BaubichonCortay H: (R)- and (S)-verapamil differentially modulate the multidrug-resistant protein MRP1. J Biol Chem 282: 31542-31548, 2007.

19. Chang XB, Hou YX and Riordan JR: ATPase activity of purified multidrug resistance-associated protein. J Biol Chem 272: 30962-30968, 1997.

20. Koski A, Raki M, Nokisalmi P, et al: Verapamil results in increased blood levels of oncolytic adenovirus in treatment of patients with advanced cancer. Mol Ther 20: 221-229, 2012.

21. Liu Y, Lu Z, Fan P, et al: Clinical efficacy of chemotherapy combined with verapamil in metastatic colorectal patients. Cell Biochem Biophys 61: 393-398, 2011.

22. Yi J, Yang J, He R, et al: Emodin enhances arsenic trioxideinduced apoptosis via generation of reactive oxygen species and inhibition of survival signaling. Cancer Res 64: 108-116, 2004.

23. Jing Y, Yang J, Wang Y, et al: Alteration of subcellular redox equilibrium and the consequent oxidative modification of nuclear factor kappaB are critical for anticancer cytotoxicity by emodin, a reactive oxygen species-producing agent. Free Radic Biol Med 40: 2183-2197, 2006

24. Igarashi T, Izumi H, Uchiumi T, et al: Clock and ATF4 transcription system regulates drug resistance in human cancer cell lines. Oncogene 26: 4749-4760, 2007.

25. Mwakigonja AR, Kaaya EE, Heiden T, et al: Tanzanian malignant lymphomas: WHO classification, presentation, ploidy, proliferation and HIV/EBV association. BMC Cancer 10: 344, 2010.

26. Mwakigonja AR, Pak F, Pyakurel P, et al: Oral Kaposi's sarcoma in Tanzania: presentation, immunopathology and human herpesvirus-8 association. Oncol Rep 17: 1291-1299, 2007.

27. Quan Z, Gu J, Dong P, et al: Reactive oxygen species-mediated endoplasmic reticulum stress and mitochondrial dysfunction contribute to cirsimaritin-induced apoptosis in human gallbladder carcinoma GBC-SD cells. Cancer Lett 295: 252-259, 2010.

28. Ding XW, Wu JH and Jiang CP: ABCG2: a potential marker of stem cells and novel target in stem cell and cancer therapy. Life Sci 86: 631-637, 2010.

29. Abaan OD, Mutlu PK, Baran Y, Atalay C and Gunduz U: Multidrug resistance mediated by MRP1 gene overexpression in breast cancer patients. Cancer Invest 27: 201-205, 2009.

30. Donmez Y, Akhmetova L, Iseri OD, Kars MD and Gunduz U: Effect of MDR modulators verapamil and promethazine on gene expression levels of MDR1 and MRP1 in doxorubicinresistant MCF-7 cells. Cancer Chemother Pharmacol 67: $823-828,2011$

31. Siddik ZH: Cisplatin: mode of cytotoxic action and molecular basis of resistance. Oncogene 22: 7265-7279, 2003. 
32. Hait WN and Yang JM: The individualization of cancer therapy: the unexpected role of p53. Trans Am Clin Climatol Assoc 117: 85-101, 2006.

33. Tazzari PL, Cappellini A, Ricci F, et al: Multidrug resistanceassociated protein 1 expression is under the control of the phosphoinositide 3 kinase/Akt signal transduction network in human acute myelogenous leukemia blasts. Leukemia 21: 427-438, 2007.

34. Meurette O, Lefeuvre-Orfila L, Rebillard A, LagadicGossmann D and Dimanche-Boitrel MT: Role of intracellular glutathione in cell sensitivity to the apoptosis induced by tumor necrosis factor \{alpha\}-related apoptosis-inducing ligand/ anticancer drug combinations. Clin Cancer Res 11: 3075-3083, 2005.
35. Cole SP, Sparks KE, Fraser K, et al: Pharmacological characterization of multidrug resistant MRP-transfected human tumor cells. Cancer Res 54: 5902-5910, 1994.

36. Cullen KV, Davey RA and Davey MW: Verapamil-stimulated glutathione transport by the multidrug resistance-associated protein (MRP1) in leukaemia cells. Biochem Pharmacol 62: 417-424, 2001.

37. Barattin R, Perrotton T, Trompier D, et al: Iodination of verapamil for a stronger induction of death, through GSH efflux, of cancer cells overexpressing MRP1. Bioorg Med Chem 18: 6265-6274, 2010.

38. Trompier D, Chang XB, Barattin R, du Moulinet D'Hardemare A, Di Pietro A and Baubichon-Cortay H: Verapamil and its derivative trigger apoptosis through glutathione extrusion by multidrug resistance protein MRP1. Cancer Res 64: 4950-4956, 2004. 